\title{
Architectonic Quantum Dot Solids
}

\author{
GIL MARKOVICH, ${ }^{\dagger}$ C. PATRICK COLLIER, ${ }^{\dagger}$ \\ SVEN E. HENRICHS, ${ }^{\dagger}$ FRANÇOISE REMACLE, \\ RAPHAEL D. LEVINE, ${ }^{\dagger}$ AND JAMES R. HEATH*,† \\ Department of Chemistry and Biochemistry, \\ University of California, Los Angeles, \\ Los Angeles, California 90095-1569, and Departement de \\ Chimie, B6, Université Liege, B 4000 Liege, Belgium
}

Received May 4, 1998

\section{Introduction}

Three things largely determine the electronic properties of a crystal: the energy levels of the atoms or lattice sites, the coupling between adjacent sites, and the symmetry of the solid. Imagine being able to control each of these properties separately, and therefore being able to "design" a solid with a prescribed set of electronic properties. For an atomic solid, this would amount to being able to tune the electronegativity of an atom, to control the strength of the covalent interactions within the lattice, and to choose the crystal structure. While the chemistry of the periodic table does not permit such control, the chemistry of "artificial atoms", ${ }^{1}$ or quantum dots (QDs), does. In this Account, we will discuss one example of "designing" a unique electronic property into a QD solid. This property, which is the ability of the solid to reversibly pass through a metal-insulator (MI) transition under ambient conditions, is something that has not been previously demonstrated for a more traditional solid. A two-dimensional (2D) hexagonal superlattice of organically passivated silver QDs was fabricated as a Langmuir monolayer. Selecting a particular size of QD controlled the (super)lattice site energies. The coupling between adjacent QDs was coarsely

Gil M arkovich is Senior Lecturer of Chemistry at Tel Aviv University. He received his Ph.D. from Tel Aviv University in 1996 and was a postdoctoral researcher at UCLA from 1996 to 1998.

C. Patrick Collier is a postdoctoral researcher at UCLA. He received a B.A. degree from Oberlin College in 1991 and a Ph.D. from the University of California, Berkeley, in 1998.

Sven E. Henrichs is currently working very hard toward a Ph.D. at UCLA. He received a B.A. from the University of Virginia in 1993.

Françoise Remacle is a "Chercheur Qualifie" of FNRS and is a member of the Laboratoire de Dynamique M oléculaire at Liege University, Belgium. Her interests are unimolecular reactions and the dynamics of excited states.

Raphael D. Levine is Max Born Professor of Natural Philosophy at the Hebrew University of $J$ erusalem and Professor of Chemistry at UCLA. His current interests include chemistry under extreme conditions including electronic processes and algebraic methods in spectroscopy.

$J$ ames R. Heath is Professor of Chemistry and Biochemistry at UCLA. His current research interests include various facets of nanoscale science, including integrated nanoscale systems for application to novel computational architectures. controlled by selection of the organic surface passivant, and precisely controlled by compressing the superlattice using the Langmuir technique. By using the title "Architectonic ${ }^{2}$ Quantum Dot Solids", we emphasize that many of the collective properties of the superlattice were rationally designed into the material.

\section{Preparation of 2D Quantum Dot Superlattices}

As wet chemical methods for the synthesis of narrow size distributions of nanoscale semiconductor ${ }^{3}$ and metal $^{4}$ QDs, or nanocrystals, have been developed, many groups have explored ways to crystallize these structures into QD superlattices. ${ }^{5}$ The silver QDs that are relevant to this Account are stabilized by covalently bound alkylthiol surface groups, and weak attractive interparticle dispersion forces drive superlattice formation. ${ }^{5,6}$ This contrasts with the entropically driven formation of artificial opals, assembled from micrometer-scale polystyrene and silica spheres. ${ }^{7}$ In many ways, the Ag QDs are the metallic equivalent of the semiconductor QDs synthesized by Bawendi's group. ${ }^{3}$ Both types of particles are highly soluble in nonpolar organic solvents and may be sizeselectively precipitated to produce size distributions of $< \pm 10 \%$ through the use of solvent/nonsolvent pairs. ${ }^{8}$ For metal QDs, interparticle interactions depend strongly on the particle size and the passivating ligand. For small $(<2$ $\mathrm{nm}$ ) particles passivated with long ligands (octadecanethiol, for example), particle aggregation is dominated by ligand-ligand interactions. ${ }^{9}$ However, for larger particles $(>3 \mathrm{~nm})$ passivated with shorter ligands (<dodecanethiol), dispersion interactions between the metal cores dominate particle aggregation, and increase in magnitude with either increasing metal core diameter or decreasing ligand length. ${ }^{5}$

At UCLA we have focused on preparing thin film QD superlattices, and have been especially concerned with quantifying the physics related to interparticle, particlesolvent, and particle-substrate interactions. We have utilized the Langmuir technique to elucidate the pressure/ temperature $(\pi / T)$ phase diagrams of monolayers of organically functionalized Ag QDs as a function of both particle size and passivating ligand. These particles can form a variety of structural phases, including foams, islands, lamellar phases, and closest packed phases. In Figure 1 we present the $\pi / T$ phase diagram, and accompanying electron micrographs, of a monolayer of $\mathrm{Ag}$ particles that compresses to form an ordered, closest packed phase.

Consider a $25^{\circ} \mathrm{C}$ isotherm through this phase diagram. At surface pressures just above $0 \mathrm{mN} / \mathrm{m}$, the monolayer is a continuous 2D polycrystalline film. The size of a single hexagonal domain ranges from 0.1 to $2 \mu \mathrm{m}$ on a side, with a typical length of $0.2 \mu \mathrm{m}$ (a few thousand particles). The monolayer in Figure 1 will compress to a surface pressure

\footnotetext{
* To whom correspondence should be addressed.

+ University of California, Los Angeles.

* Université Liege.
} 


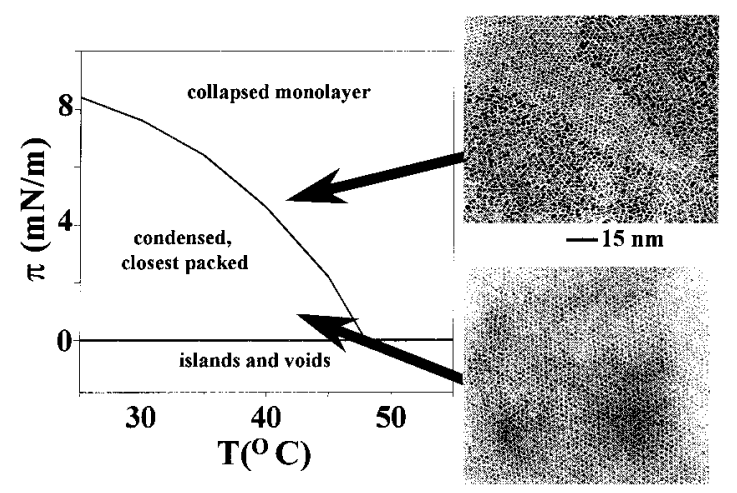

FIGURE 1. Langmuir phase diagram and corresponding TEM images for a monolayer of decanethiol-passivated, $30 \AA$ silver nanocrystals.

of $\sim 8 \mathrm{mN} / \mathrm{m}$ at $25^{\circ} \mathrm{C}$ before it collapses into a bilayer. During this compression range, the interparticleseparation distance decreases, and so experiments aimed at probing the properties of the monolayer can be carried out as a continuous function of interparticle separation distance. Throughout this Account, we will refer to interparticle separation distance as either the distance between the surfaces of adjacent metallic spheres, $\delta$, or as the particle size-normalized separation, $D / 2 r$. D is the separation between particle centers, and $r$ is the radius of the metallic core. Dilute solutions of particles are represented by some limiting large $D / 2 R$ value. Ag particles of $3 \mathrm{~nm}$ diameter capped with dodecanethiol can typically be compressed from $\delta=2 \mathrm{~nm}$ to $\delta=1.0 \mathrm{~nm}( \pm 0.2 \mathrm{~nm})$, or from $\mathrm{D} / 2 \mathrm{r}$ of 1.7 to $\mathrm{D} / 2 \mathrm{r}$ of 1.3 . For $3 \mathrm{~nm} \mathrm{Ag}$ particles capped with propanethiol, $\delta$ may be varied from about 0.8 to about $0.4 \mathrm{~nm}$. These numbers fluctuate somewhat from synthesis to synthesis. Although shorter-chain ligands correspond to smaller interparticle separation distances, so do fewer ligands (lower surface coverage). Particles that have been precipitated many times will have a low surface coverage.

In Figure 2 we present color images of a monolayer of butanethiol-passivated $3 \mathrm{~nm}$ diameter Ag QDs. The QDs are characterized by a very strong (collective) resonance that gives the film its deep maroon color. The top image of Figure 2 is of particles that have been compressed to a $D / 2 r$ value just below 1.2. In the bottom image, $\delta$ has been slightly reduced (by about 5\%), and the film now has a metallic, silvery luster which is seen spanning the centerline of the film. The metallic luster appears at, or possibly just after, the onset of the MI transition. Before we discuss how the properties of our monolayers vary as a function of $D / 2 r$, we first provide some background for the MI transition, and how it was "designed" into our system.

\section{Physics of Coupled Metal Quantum Dots}

Superlattices of metal QDs are a special case of matter. Although they mainly consist of metal, their electronic properties are generally different from those of bulk metals. The separation of energy levels in a metal QD is on the order of $\epsilon_{\mathrm{f}} / \mathrm{N}$. Here $\epsilon_{\mathrm{f}}$ is the Fermi energy of the metal and $\mathrm{N}$ is the number of atoms in the metal. ${ }^{10}$ For a
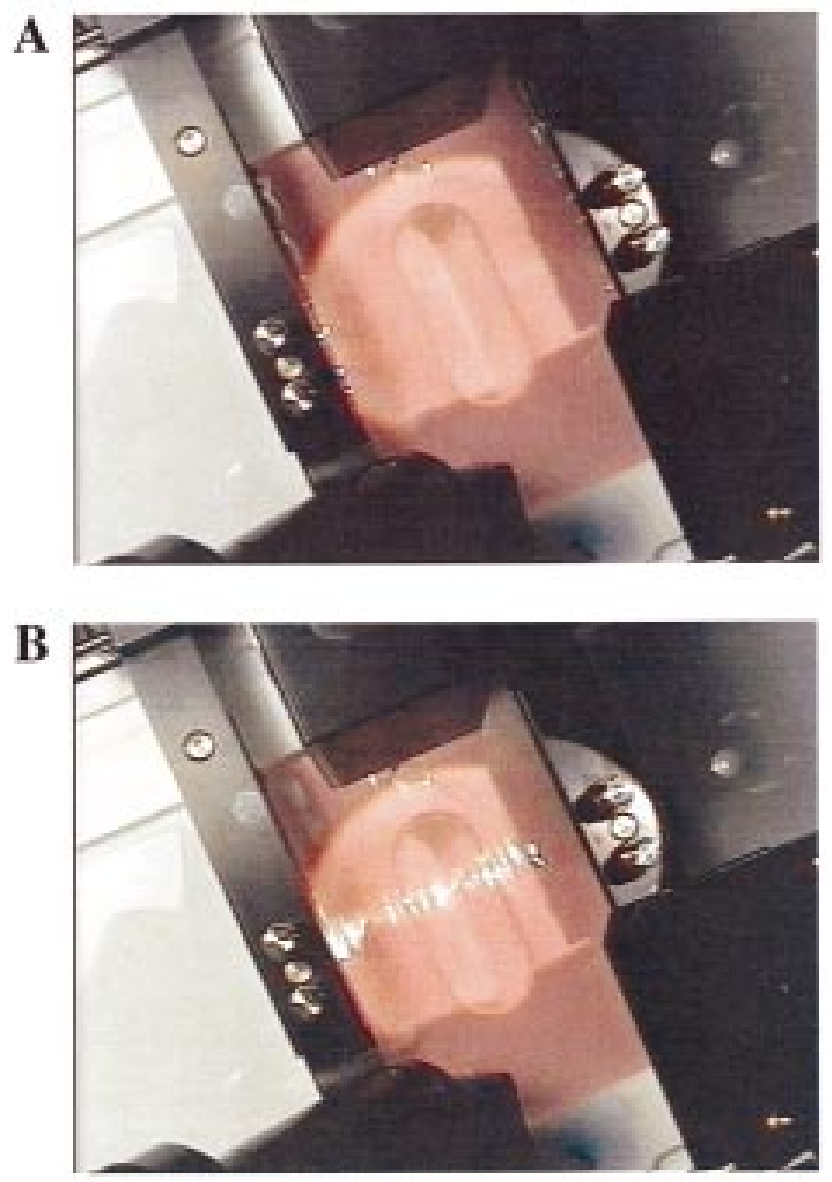

FIGURE 2. Photographs taken during compression of a Langmuir monolayer of butanethiol-capped, $35 \AA$ diameter silver particles, before (a) and just after (b) the metal-insulator transition.

$30 \AA$ diameter $\mathrm{Ag}$ particle ( $\sim 10^{3}$ atoms), this number is 5-10 meV. ${ }^{11}$ Given that $\mathrm{kT}$ at $300 \mathrm{~K}$ is about $25 \mathrm{meV}$, this implies that the energy levels in a $30 \AA$ particle are essentially continuous, and that the particle is "metallic". However, unlike bulk metal, a nanocrystal is characterized by a size-dependent finite capacitance, $C(r)=4 \pi \epsilon_{0} \in r$, where $r$ is the radius of the particle, $\epsilon_{0}$ is the permittivity of vacuum, and $\epsilon$ is the dielectric constant of the material surrounding the particle. Because of this, the particle is characterized by a charging energy, $\mathrm{U}_{\mathrm{c}}=\mathrm{e}^{2} / \mathrm{C}$, which is the energy required to remove an electron from one isolated dot and add it to another isolated dot. Here e is the fundamental unit of charge. ${ }^{12,13}$ For a $30 \AA$ diameter particle surrounded by a material with $\epsilon=3, C(r)=5 \times$ $10^{-19} \mathrm{~F}$, and $\mathrm{U}_{\mathrm{c}}$ is about $0.3 \mathrm{eV}$.

$U_{c}$ will govern the electrical properties of a lattice of metal QDs. Such a lattice is a good model system for the Hubbard-type Hamiltonian, ${ }^{14}$ in which correlation due to repulsive Coulombic interactions between electrons tends to localize charges at distinct lattice sites. In an atomic solid, the intra-atomic Hubbard energy, $U$, is the energy required to take an electron from one site and put it on another site, already occupied by an electron. This is exactly analogous to the definition of $U_{c}$ for a metal nanocrystal. In the limit of infinite separation between atoms (or dots), it is then the difference of the ionization 


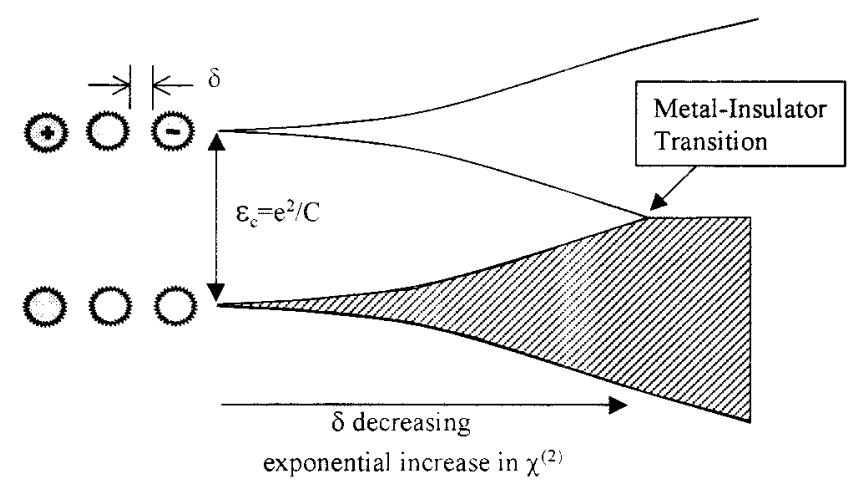

FIGURE 3. Representation of the effects of interparticle separation distance on the electronic structure of the QD superlattice. The energy to separate a positive and a negative charge carrier is the width of the Coulomb gap for a single particle, and is equal to $e^{2} / C$. As interparticle separation is reduced, exchange interactions lead to the development of energy bands, and eventually the formation of a metallic solid.

potential and the electron affinity of the atom (or dot)

$$
U=I P-E A=U_{c}
$$

For a lattice of weakly interacting QDs, the fact that each lattice site is characterized by a finite charging energy means that there is a Coulomb gap of width $U$ in the electronic band structure of the (artificial) solid, effectively making the superlattice an insulator. This gap is a particlesize-dependent barrier to current flow, or charge delocalization, and the ability to tune this gap by changing particle size is a major aspect of this work. The fact that our superlattice has a Coulombic gap makes it conceptually similar to an atomic (insulating) solid, and separates it from much previous work on metal particles and islands. We will revisit this point later.

We now consider interdot coupling. When two metal QDs are far apart from one another $(\delta=1-2 \mathrm{~nm}$, for example), the electric fields of the particles will couple via classical, or multipole, coupling. Any charge carriers are either injected or thermally excited, and electrical conductivity proceeds by activated (phonon-assisted) hopping. At low temperatures, activated hopping is quenched, and injected carriers can tunnel through the lattice. For $\delta<10 \AA$, the quantum mechanical wave functions of adjacent particles will begin to overlapleading to what is known as exchange coupling. As the strength of exchange coupling increases, the kinetic energy of the particle wave functions is reduced (similar to how a chemical bond is formed), and this will, in turn, begin to overcome the site charging energy, and the Coulomb gap decreases. ${ }^{15}$ Upon further reduction of $\delta$, the gap closes, and the solid will undergo a Mott-Hubbard MI transition. Consequently, at large enough interdot coupling our artificial solid is expected to attain metallic-like properties. This transition is shown schematically in Figure 3. This transition is not a percolation transition. For a percolation transition, a tunneling barrier between grains or particles may be present, but no Coulomb band gap is implied. At the percolation threshold, at least a single continuous conduction pathway through the system is established. For our system, at the MI transition, the Coulomb gap disappears.

\section{Characterization of Quantum Dot Coupling and the MI Transition}

As we compress the monolayer, we perform up to six distinct measurements as a continuous function of interparticle separation distance (Figure 4). The six measurements are (1) surface pressure, (2) UV/ vis reflectance and (3) transmission, (4) second harmonic generation (SHG), (5) frequency-dependent complex impedance, and (6) a video movie of the experiment. In addition, we have transferred films to solid supports and measured optical properties, film structure by TEM or scanning tunneling microscopy (STM), and temperature-dependent tunneling spectroscopy. ${ }^{16}$

The physics described in the previous section should be applicable for any variety of metal QDs. Silver QDs, however, are experimentally advantageous in that they can be prepared with a narrow size distribution, they have reasonable chemical stability, and they are characterized by a surface plasmon (collective) resonance, $\omega_{\text {sp }}$, in the visible. This resonance, which has an oscillator strength that scales as the particle volume, ${ }^{17}$ makes the monolayers easily visible by the eye. $\omega_{\text {sp }}$ is sensitive to the local fields surrounding the particle, which means that both the linear and the nonlinear optical properties are excellent probes of interparticle coupling. ${ }^{18,19}$ The linear and nonlinear optical signatures essentially provide information relevant to how the high-frequency dielectric function of the superlattice is modified as interparticle coupling strength is increased. However, as the system is compressed from an insulator to a metal, the mechanism of charge transport changes, and that mechanistic information is contained in the low-frequency response of the superlattice. Thus, we also measure the complex impedance of the superlattice from $10 \mathrm{kHz}$ to $10 \mathrm{M} \mathrm{Hz}$, as a function of compression. ${ }^{20}$ Finally, we utilized STM-based DC measurements to quantify how the electronic density of states (DOS) of the superlattice changes. This last measurement indicates how the electronic structure of the superlattice rehybridizes, and temperature-dependent DOS measurements yield information relevant to how the Coulombic band gap is affected by interparticle separation distance. In the following sections, we discuss each of these measurements separately, before piecing the whole picture back together.

\section{The Linear Optical Properties}

We discuss the optical properties of monolayers of $3 \mathrm{~nm}$ Ag QDs as functions of $D / 2 r$. As $D / 2 r$ is decreased from a limiting solution-phase value to that of the compressed superlattice with $D / 2 r \cong 1.2, \omega_{\text {sp }}$ shifts to lower energy and the reflectivity at normal incidence can increase by as much as 6 times. These changes are well described by a classical effective medium model that accounts for the changing local electric fields around a particle. ${ }^{21}$ This model predicts increasing reflectivity until $D / 2 r$ reaches the minimum value of 1 . However, between $D / 2 r=1.2$ 


\section{Optical and Transport Measurements Correlated to}

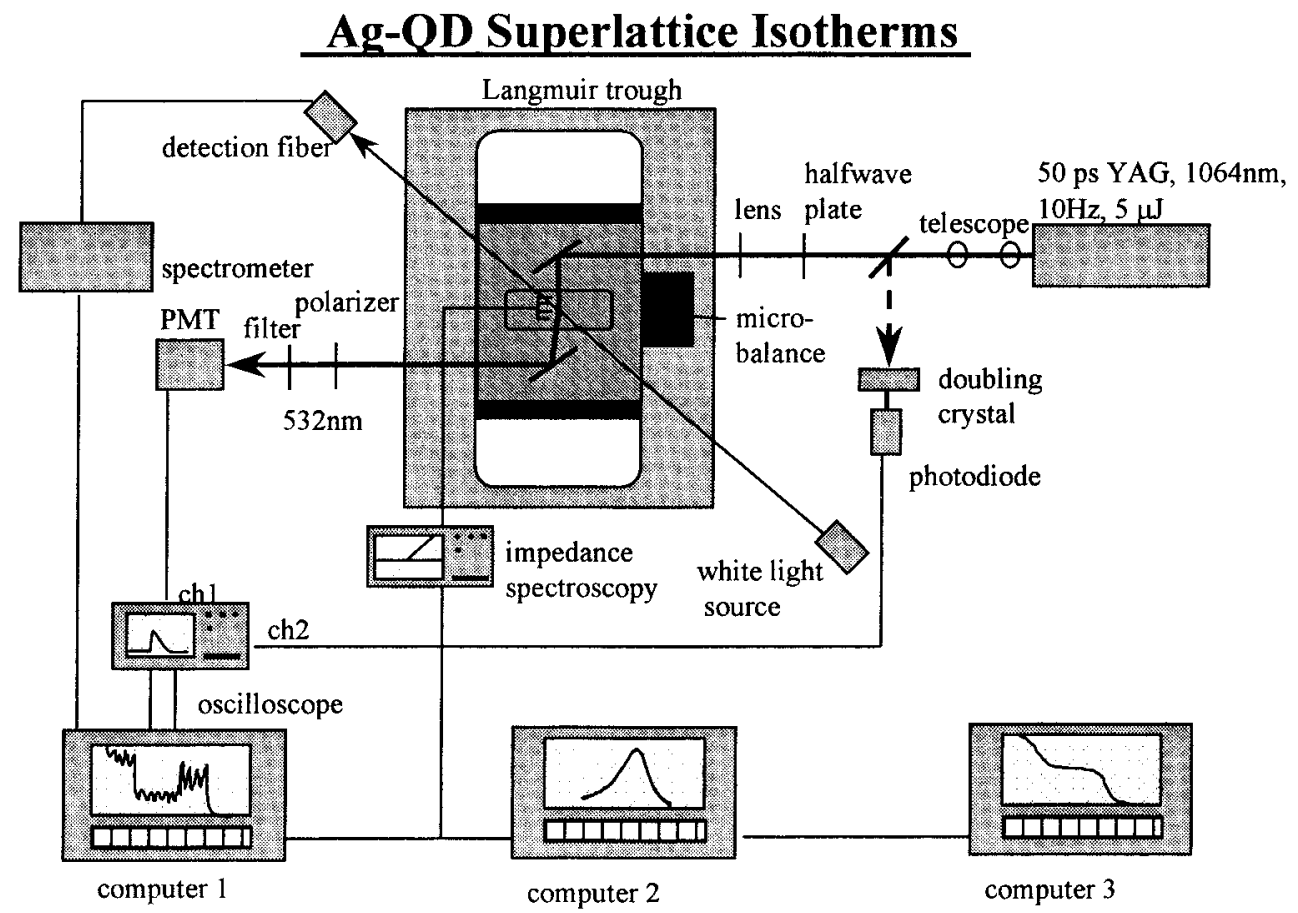

FIGURE 4. A schematic of the experimental setup used for all but the tunneling spectroscopy measurements discussed here.

and $\mathrm{D} / 2 \mathrm{r}=1.1$ the amplitude of the optical reflectivity sharply decreases, and the signature of $\omega_{\mathrm{sp}}$ almost disappears from the recorded reflectance spectrum. The final reflectance spectrum resembles that of evaporated silver island films at their reported percolation threshold. ${ }^{22} \mathrm{We}$ thus assign the sharp decrease in reflectance in our case to a MI transition. The reversibility of this transition can be probed by measuring the reflectance as the trough barriers are expanded, and then recompressed (Figure 5). Consequently, we believe that the drop in reflectivity correlates with a reversible MI transition. ${ }^{23,24}$

To obtain a quantitative description of these optical changes, we have inverted reflectivity and transmissivity data taken simultaneously at normal incidence to deduce values for the complex dielectric function, $\mathrm{n}^{2}=\epsilon(\omega)=$ $\epsilon_{1}(\omega)+\mathbf{i} \epsilon_{2}(\omega)$, for film compression states up to the MI transition. We used a well-established matrix method 25 to calculate $\epsilon(\omega)$ appropriate for a thin absorbing film sandwiched between two transparent layers (air and water). The values of the complex dielectric functions presented in Figure 6 reproduce the experimental $R$ and $T$ values to within a fraction of $1 \%$.

The measured optical properties of our QD monolayers have two possible solutions (Figure 6) for $\epsilon(\omega)$, for wavelengths in the near-IR to near-UV range. ${ }^{26}$ The solution with the positive-valued real part of $\epsilon(\omega)$ is appropriate for an insulator, and should be more physically realistic for the uncompressed films. This solution agrees well with $\epsilon(\omega)$ calculated from a classical effective medium model of the silver QD superlattices that included dipole (including many-body) coupling between nano-
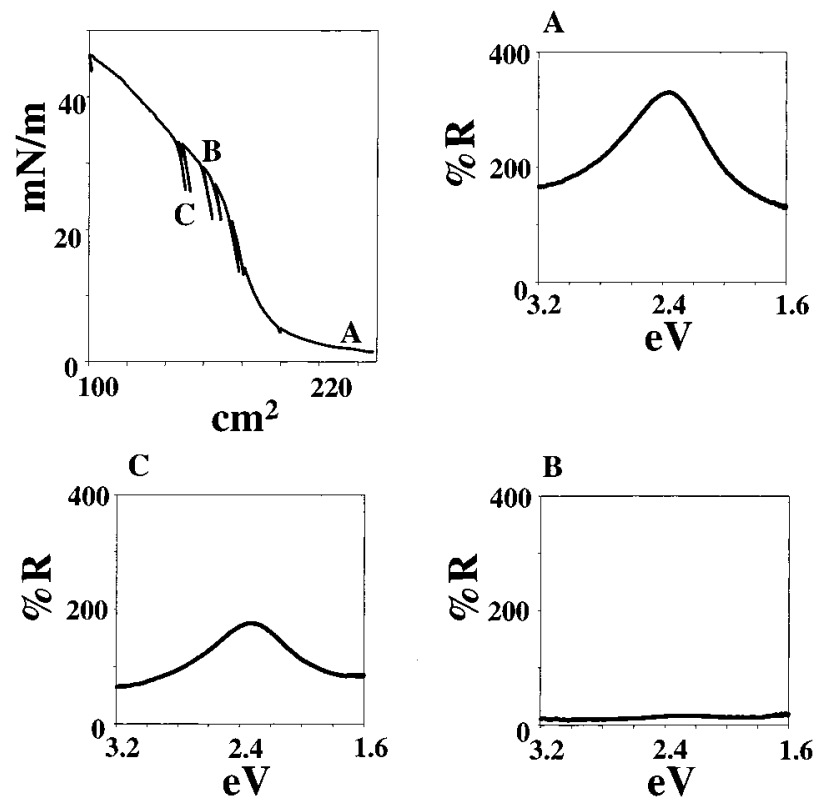

FIGURE 5. Normal incidence reflectance data showing the metalinsulator transition and the optical switching capabilities of $\mathrm{Ag} Q D$ monolayers. At top left is a $\pi / A$ isotherm for the compression of propanethiol-capped, $30 \AA$ particles with three labels corresponding to the spectra. Spectrum $A$ is reflection from an uncompressed monolayer, relative to that of a pure water surface. Spectrum $B$ is taken after compression through the MI transition. $C$ was taken after the monolayer was reexpanded to an insulator.

particles. ${ }^{21}$ The negative-valued solution to the real part of $\epsilon(\omega)$ appears as a mirror image to the positive-valued solution.

At the MI transition, the dispersion of the real part of $\epsilon(\omega)$ changes sharply while the imaginary part is relatively 


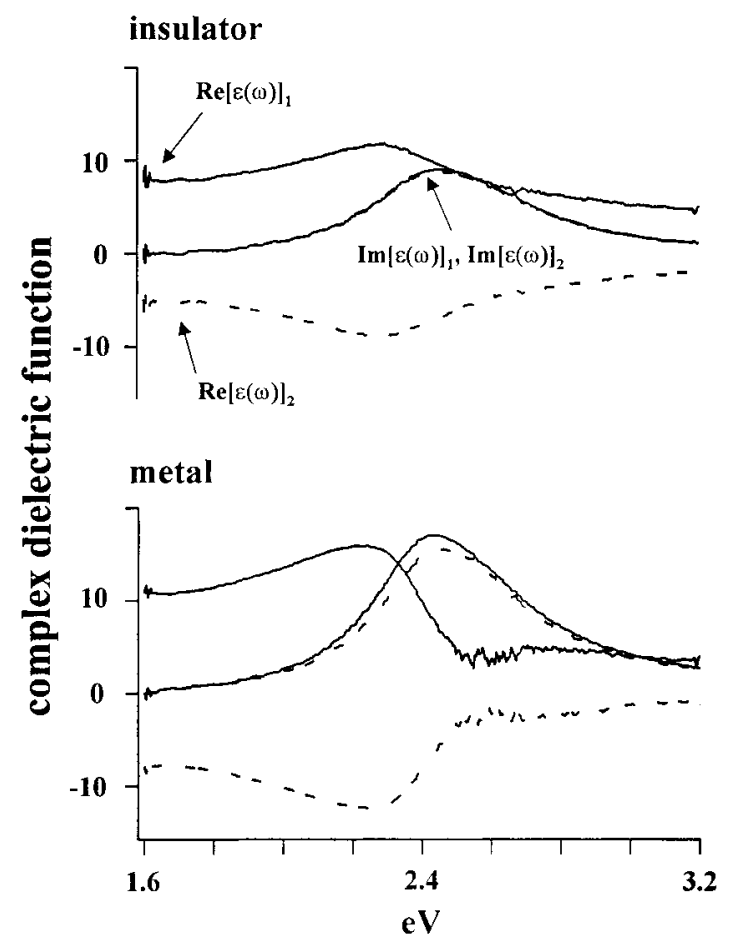

FIGURE 6. Values of the complex dielectric function on either side of the metal-insulator transition, deduced from normal incidence reflectivity and transmissivity measurements. Both possible solutions are shown; see the text for details.

unaffected. The positive- and negative-valued solutions to the real component of $\epsilon(\omega)$ approach each other and with further compression become a single solution at around $2.5 \mathrm{eV}$. At this point, the negative-valued solution for the real part of $\epsilon(\omega)$ may be the physically realistic choice. This is consistent for a metallic film. Nearly identical behavior has been reported at the percolation threshold for silver island films at high metal volume fraction. ${ }^{22}$ This correspondence implies that the final state for both systems is at least a quasi-continuous metallic film. The similarity in the optical properties of these two different systems highlights the fact that the highfrequency dielectric response of the QD monolayers is not sensitive to the presence of a Coulomb gap. For the case of evaporated $\mathrm{Ag}$ islands that are grown in size up to the percolation threshold, the island size, just below the threshold, is around $0.1 \mu \mathrm{m}$. Those islands have a charging energy of $\sim 10-20 \mathrm{meV}$, which is less than $\mathrm{kT}$ at $300 \mathrm{~K}$. An array of such islands has no Coulomb gap. The optical signature of the percolation threshold, or of our MI transition, is that of isolated islands, or QDs, transforming to a quasi-continuous metallic film.

\section{The Nonlinear Optical Response}

The nonlinear optical response of Ag QD monolayers provides information that complements that provided by the linear optical response. First, it is possible to extract a single, material-dependent parameter-the second-order polarizability $\left(\chi^{(2)}\right)$ - of the superlattice. A measurement of how this property changes as a function of interparticle separation should reveal the functional form of the interparticle coupling. Second, $\chi^{(2)}$ is sensitive to the local symmetry of the monolayer-something that the linear optical properties do not sample. Order within the superlattice is a particularly important issue with respect to the MI transition, and the $\chi^{(2)}$ measurement gives us some insight into the importance of order.

In the dipole approximation, a nonzero $\chi^{(2)}$ requires a lack of inversion symmetry. ${ }^{27}$ The air/superlattice/ water interface of our system is, of course, asymmetric with respect to inversion. Within the plane of the superlattice, variations in particle size and separation distances also provide a mechanism for symmetry breaking. Resonance enhancement, due to the strong spectral overlap of $\omega_{\mathrm{sp}}$ with the second harmonic of our pulsed YAG laser (532 $\mathrm{nm}$ ) generated by the films, ensures that changes in $\chi^{(2)}$ are nearly background free measurements. Furthermore, a nonlinear optical response is enhanced if the resonant state has high oscillator strength and large volume,28 criteria that are satisfied by Ag QDs.

Second harmonic generation (SHG) experiments were carried out by frequency doubling a 30 ps, 1064 nm laser beam off the surface of the QD monolayer and recording the intensity of $532 \mathrm{~nm}$ light as a function of compression. The SHG signal increased by a factor of $\sim 500$ as $3 \mathrm{~nm}$ particles were compressed from $\mathrm{D} / 2 \mathrm{r}=1.4$ to below $\mathrm{D} / 2 \mathrm{r}$ $=1.2$. This response depends on changes in the linear optical properties, as well as changes in $\chi^{(2)}$. 27 Since we had separately measured the linear optical properties, we were able to isolate changes in $\chi^{(2)}$, and showed that $\chi^{(2)}$ increased exponentially with respect to decreasing $D / 2 r$. An exponential increase, as opposed to a geometric increase (such as $r^{-6}$ ), implies that exchange coupling is important for values of $\delta<10 \AA$.

Remacle et al. ${ }^{29}$ utilized a 1-electron Hamiltonian within the context of a tight binding model to calculate the change in $\chi^{(2)}$ as a function of $\mathrm{D} / 2 \mathrm{r}$ for a single hexagonal superlattice domain containing $\sim 500$ particles. They calculated an increase of the SHG only after introducing small $(\leq 10 \%)$ random fluctuations to both the particle site energies (compositional disorder) and their coordinates (configurational disorder). These random fluctuations, which agree with experiment, provide for symmetry breaking in the plane of the superlattice, yielding an exponentially increasing $\chi^{(2)}$ with decreasing $\mathrm{D} / 2 \mathrm{r}$. For nonequivalent lattice sites, charge carriers will localize at the lowest energy sites. At some interparticle separation distance, exchange coupling overwhelms the effects of disorder. The system becomes "effectively" ordered, and $\chi^{(2)}$ is calculated to drop. The computed broad peak in $\chi^{(2)}$ in Figure 7 occurred at a transition from a system of disorder-driven, localized electronic states to a band-like delocalized electronic structure. The peak in the experimental measurement of Figure 7 (near $D / 2 r \approx$ 1.15) is much sharper, perhaps indicating that something more than a disorder/order transition, such as the MI transition, is observed experimentally. The drop in the experimental SHG measurement does correspond to the optical signature of the MI transition. The theory of 


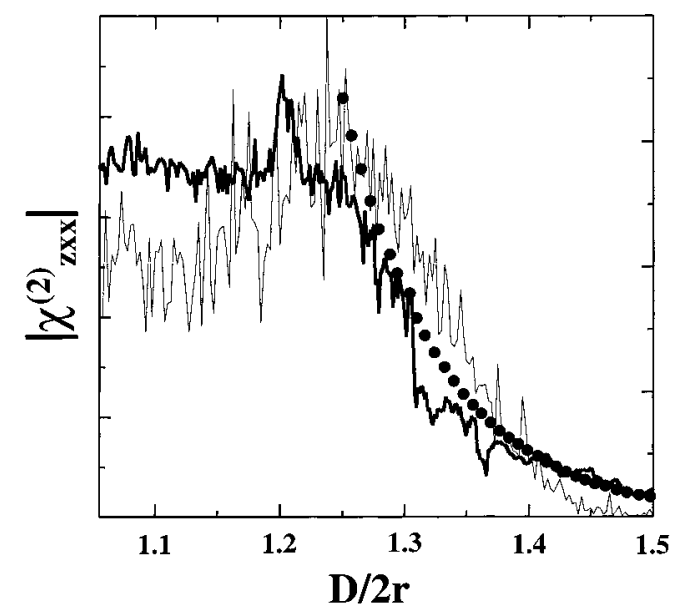

FIGURE 7. Experimental measurement (bold line) and 1-electron model calculation for the SHG generation from compressed superlattices.

Remacle et al. breaks down in the limit of strong exchange coupling, and therefore would not have detected a MI transition.

\section{Frequency-Dependent ac Impedance Measurements}

In this section we discuss low-frequency transport measurements that are sensitive to the electronic character of interdot coupling. Consider our 2D array of QDs as an electrical circuit. Each dot has a finite capacitance, and is separated from every other dot by an organic medium, which serves as a tunneling barrier. The equivalent electrical circuit is that of a large, $2 \mathrm{D}$ array of capacitively coupled tunnel junctions, or an RC circuit. Using ac impedance spectroscopy (IS), 20,30 it is possible to separate the contributions from the water subphase to the signal, and directly analyze the complex impedance of the monolayer, as a continuous function of $D / 2 r .{ }^{30}$

Measurements were carried out on propanethiol-passivated $35 \AA$ diameter silver QD monolayers as a function of compression, and correlated with optical reflectance measurements.

There are several variations of complex impedance spectroscopy, and after much trial and error, we settled on measuring the complex admittance $\left(Y^{*}\right)$, which is the inverse of the complex impedance. Two organically functionalized Pt electrodes are brought into contact with the monolayer, and an ac voltage signal, tunable from $10 \mathrm{kHz}$ to $10 \mathrm{MHz}$, is applied across the electrodes. An impedance analyzer uses a double null detector to determine the phase and amplitude changes in the applied signal. The net film admittance data $\left(Y^{*}\right)$ are then transformed to the complex dielectric modulus representation as $\mathrm{M}^{*}=\mathrm{jC}_{0} \omega /$ $Y^{*}$. Here, $j=\sqrt{ }-1, C_{0}$ is the geometric capacitance of the cell defined by the electrodes and film, and $\omega$ is the angular frequency. The dielectric modulus is the inverse of the complex dielectric permittivity, and is a particularly useful functional form to plot the impedance data when conductive systems with relatively low capacitance are

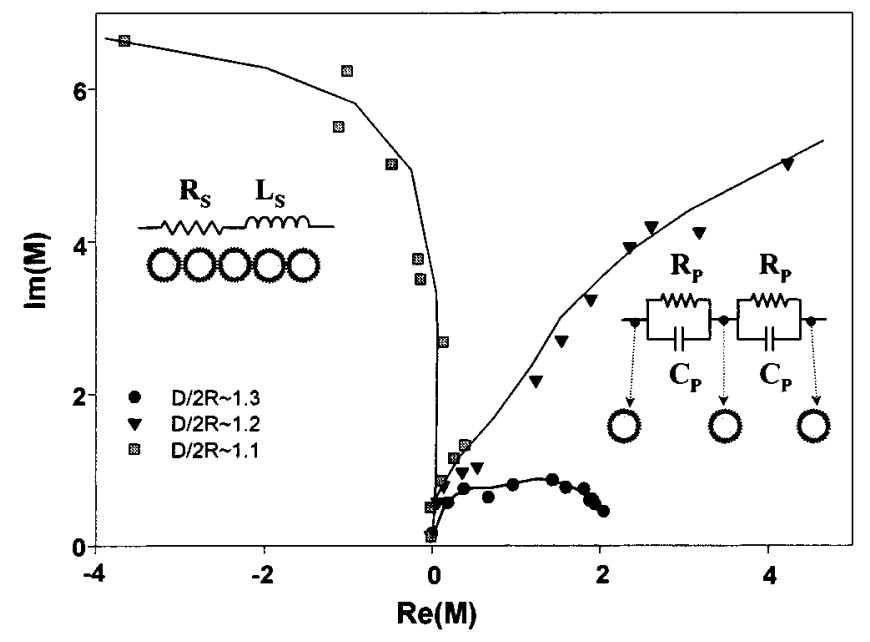

FIGURE 8. The results of AC impedance spectroscopy taken during film compression. The semicircles at low compression are indicative of capacitive tunneling interactions between particles. As $D / 2 r$ decreases, the real part of the dielectric modulus switches to negative, indicating the onset of inductive behavior, a signature of metallic transport.

studied. ${ }^{31}$ Recall that metal QDs in this size regime typically have a capacitance of $10^{-19}$ to $10^{-18} \mathrm{~F}$ per particle. ${ }^{32}$

Experimental results are displayed in Figure 8. At low compression, we observe distorted semicircles in the complex modulus plane. A single semicircle can be modeled by an equivalent parallel RC circuit, 33 where $\tau$ $=\mathrm{RC}$ is the dielectric relaxation time. Disordered systems are typically better described by a distribution of RC values. ${ }^{31}$ Since our particle films are reasonably well ordered, we expect a narrower distribution of relaxation times. By fitting the experimental curves on the positive side of the real $\mathrm{M}$ axis, we obtain $\tau$ for different compression states of the films.

The fitted relaxation time sharply decreases at the last part of the compression, when $\mathrm{D} / 2 \mathrm{R}<1.3$. In this range of interparticle separation, the interdot coupling increases exponentially with compression and the decrease in relaxation time correlates with an increase in tunneling rates between nanocrystals.

As $D / 2 r$ approaches a value of 1.1 , a sharp change in the complex modulus plots is observed. As can be seen in Figure 8, the curves shift from the positive side of the real $M$ axis to its negative side. This implies that the film has become inductive (left side of Figure 8 ). The inductive current is $180^{\circ}$ off the capacitative current phase, and should therefore contribute negatively to the real part of $M *$. The $D / 2 r \approx 1.1$ curve in Figure 8 was fitted to a series $\mathrm{RL}$ circuit, to represent the film inductance.

Inductive transport is a characteristic of metallic conduction. Alternating current creates a time-varying magnetic field, which reacts with the charge carriers to change the current phase/amplitude relation to the exciting voltage. The charge carriers will produce a significant magnetic field only while maintaining their velocity vector over large distances. This is possible only with coherent transport and not with local hopping or tunneling trans- 
port in which electron motion is random on a short length scale. The switch from RC type tunneling transport to coherent, long-range transport is typical of insulator-tometal transitions, and strengthens the deductions from the optical data.

\section{Tunneling Spectroscopy on Transferred Films}

All experiments discussed thus far have investigated the statistical (area-averaged) properties of our QD monolayers as a function of compression. In this section, we look at how the electronic structure of single QDs is effected as the monolayer passes through the MI transition. We utilized a variable temperature STM as a technique for carrying out DC transport measurements through individual QDs in a monolayer, at a few fixed values of $\delta .^{16}$ The normalized differential conductance is a measurement of the DOS of a solid, and therefore can probe the presence or absence of a Coulombic gap, or more generally, insulating or metallic behavior. For these experiments, monolayers of $\sim 3 \mathrm{~nm}$ diameter silver QDs, passivated with decanethiol and hexanethiol, were prepared on a Langmuir trough and transferred to highly oriented pyrolytic graphite substrates for STM analysis. For the hexanethiol-capped particles, the optical properties of the monolayer indicated that it was metallic prior to film transfer. In Figure 9 we present the temperature-dependent normalized DOS for decanethiol (top) and hexanethiol (bottom) capped particles. Pentanethiol-capped particles exhibited a DOS nearly identical to that of the hexanethiolcapped particles. For the decanethiol-capped particles, we found a strongly temperature-dependent DOS at $0 \mathrm{~V}$ that tends to 0 at low temperatures. This is the Coulomb gap. For the hexanethiol-capped QD monolayer, we found strong evidence for rehybridization of the electronic structure of the superlattice. The normalized DOS has a temperature-independent value of 1 at $0 \mathrm{~V}$. This is the signature of a metallic film. These experiments not only provide further confirmation of the MI transition, but also indicate that the electronic properties of the films are retained even after they are transferred from the water surface.

\section{Summary}

Following the above experiments, we now outline our concept of the changes in electronic properties of the QD superlattices as interparticle separation distance, $\delta$, is reduced. For $\delta>1 \mathrm{~nm}$, electronic states are localized on the individual QDs, although the QDs are strongly dipolecoupled to one another. Localization arises from the presence of the Coulomb gap, and from disorder in the superlattice. At $300 \mathrm{~K}$ and large $\mathrm{D} / 2 \mathrm{r}$, the conductivity is dominated by thermally activated hopping. Throughout this weak-coupling regime the reflectivity of the film increases, and $\omega_{\mathrm{sp}}$ red shifts with decreasing $\mathrm{D} / 2 \mathrm{r}$. The exponential increase in $\chi^{(2)}$ occurs as $\delta$ is decreased from 1 to $0.6 \mathrm{~nm}$. Throughout this regime, charge tunneling and

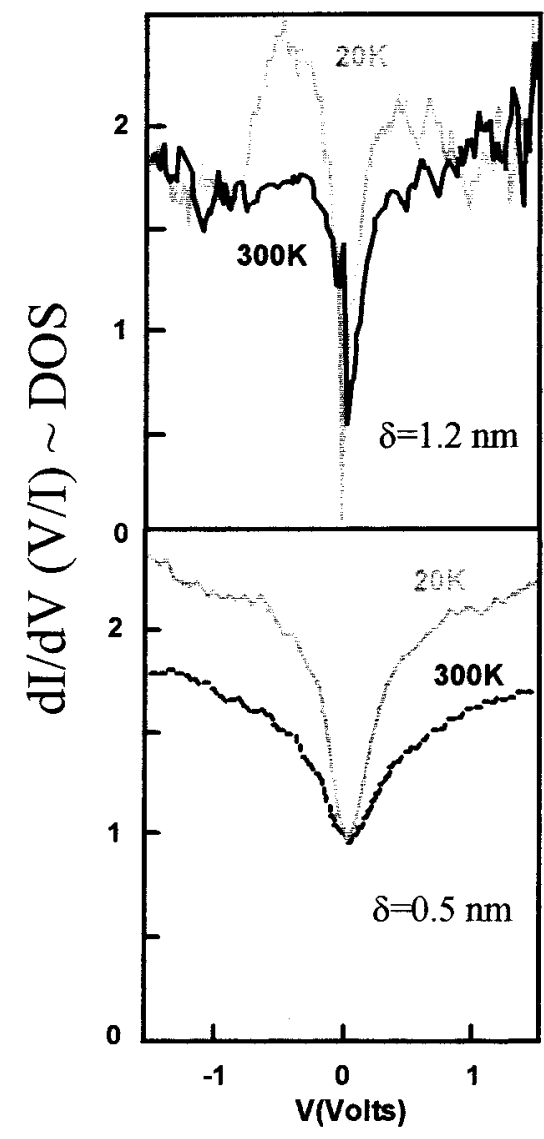

FIGURE 9. Normalized density of states (DOS) measurements for dec anethiol (top) and hexanethiol (bottom) passivated Ag QDs. Each curve represents an average of a few hundred measurements. The DOS at $20 \mathrm{~K}$ for the decanethiol-capped particles tends toward zero at $0 \mathrm{~V}$, consistent with the presence of a Coulomb gap. In the bottom curves, the DOS at $0 \mathrm{~V}$ is temperature-independent. A normalized DOS value of 1 at $0 \mathrm{~V}$ is a signature of a metallic film.

exchange interactions are important. The characteristic (tunneling) time constant of the film decreases throughout this region.

The increase in tunneling coupling continues with compression until $\mathrm{D} / 2 \mathrm{r}$ is near 1.1 , at which point exchange interactions overcome the Coulomb gap. Charge carriers can then be generated with no cost in energy, and according to the Mott criterion for a metal-insulator transition, ${ }^{23}$ about one electron per dot becomes fully delocalized, to form a metallic state of matter. This transition to a metal is observed in all probes: the optical properties abruptly change; $\chi^{(2)}$ drops sharply; the film becomes inductive; and a temperature-independent metallic DOS is observed at the Fermi level.

The role of order-disorder to the MI transition for our system of QDs has yet to be quantified. We have investigated monolayers that are characterized by broad particle size distributions, and although the reflectance spectra of such films indicate strong dipole-dipole coupling, we have never observed an MI transition in such films. Nevertheless, even our "best" films exhibit some disorder. The (Coulombic) site-charging energy that characterizes our "artificial atoms" of silver QDs is large $\left(U_{C}\right.$ $\approx 0.3 \mathrm{eV}$ ) relative to the expected single electron energy 
level spacing within a single particle ( $\sim 5 \mathrm{meV})$, and makes our system unique within the context of MI transitions. Since the Coulomb gap is $\gg k_{B} T$ at room temperature, this system should be well below the critical temperature for the existence of a first-order metal-insulator transition. ${ }^{23}$

\section{Concluding Remarks}

These architectonic solids provide an opportunity to study a variety of fundamental physics, of which the MI transition is just one aspect. One direction of studies is the exploration of scaling laws by investigating other lower or higher dimensional structural phases of nanocrystals, such as "rings", "wires", ${ }^{34}$ or 3D superlattices. Alternatively, it should be possible to tune other quantum interactions, such as ferromagnetic and Josephson exchange. Although we have not discussed applications here, it should be possible to use QD solids as highly nonlinear and sensitive sensors or optical switches, to name just two possibilities. The key is that with QD solids, it is possible to control the fundamental properties of a solid with a precision that has been previously inaccessible.

We acknowledge R. Saykally, C. Knobler, W. Gel bart, R. Shen, J. Shiang, S. Williams, G. Madeiros-Ribeiro, and D. Ohldberg. J.R.H. acknowledges support from the ONR, the NSF, a Packard Fellowship, and a Sloan Fellowship.

\section{References}

(1) Kastner, M. A. Artificial Atoms. Phys. Today 1993, 46, 24. Ashoori, R. C. Electrons in Artificial Atoms. Nature 1996, 379, 413.

(2) Architectonic: having an organized and unified structure that suggests an architectural design (Merriam-Webster's WWW dictionary). Dr. R. S. Williams, Hewlett-Packard Labs, suggested this term.

(3) Murray, C. B.; Kagan, C. R.; Bawendi M. G. SelfOrganization of CdSe Nanocrystallites into ThreeDimensional Quantum Dot Superlattices. Science 1995, 270 , 1335.

(4) Brust, M.; Walker, M.; Bethell, D.; Schiffrin, D. J.; Whyman, R. Synthesis of Thiol-Derivatised Gold Nanoparticles in a 2-Phase Liquid-Liquid System. J. Chem. Soc., Chem. Commun. 1994, 801.

(5) Collier, C. P.; Vossmeyer, T.; Heath, J. R. Quantum Dot Superlattices. Annu. Rev. Phys. Chem. 1998, 49, 371.

(6) Hamaker, H. C. The London-van der Waals Attraction Between Spherical Particles. Physica 1937, 4, 1058.

(7) Pusey, P. N.; Poon, W. C. K.; Ilett, S. M.; Bartlett, P. Phase Behavior and Structure of Colloidal Suspensions. J. Phys. Condens. Matter. 1994, 6, A29.

(8) Harfenist, S. A.; et al. Three-Dimensional Hexagonal Close-Packed Superlattice of Passivated Ag Nanocrystals. Adv. Mater. 1997, 9, 817.

(9) Luedke, W. D.; Landman, U. Structure, Dynamics, and Thermodynamics of Passivated Gold Nanocrystals and Their Assemblies. J. Phys. Chem. 1996, 100, 13323.

(10) Kubo, R. Electronic Properties of Metallic Fine Particles. J. Phys. Soc. Jpn. 1962, 17, 975.

(11) Schaaff, T. G.; et al. I solation of Smaller Nanocrystal Au Molecules: Robust Quantum Effects in Optical Spectra. J. Phys. Chem. B 1997, 101, 7885.
(12) Lambe, J.; Jaklevic, R. C. Charge-Quantization Studies Using a Tunnel Capacitor. Phys. Rev. Lett. 1969, 22, 1371 .

(13) Cavicchi, R. E.; Silsbee, R. H. Dynamics of Tunneling to and from Small Metal Particles. Phys. Rev. B 1988, 37, 706.

(14) Hubbard, J. Electron Correlations in Narrow Energy Bands III. An Improved Solution. Proc. R. Soc. London, A 1964, 281, 401.

(15) (a) Stafford, C. A.; Das Sarma, S. Collective Coulomb Blockade in an Array of Quantum Dots - A MottHubbard Approach. Phys. Rev. Lett. 1994, 72, 3590. (b) Abeles, B.; Sheng, P.; Coutts, M. D.; Arie, Y. Structural and Electrical Properties of Granular Metal Films. Adv. Phys. 1975, 24, 407 and references therein.

(16) Medeiros-Ribeiro, G.; Ohldberg, D.; Williams, R. S.; Heath, J. R. Rehybridization of Electronic Structure in Compressed 2D Quantum Dot Superlattices. Phys. Rev. B, in press.

(17) (a) Bohren, C. F.; Huffman, D. R. Absorption and Scattering of Light by Small Particles; Wiley: New York, 1983. (b) Kreibig, U.; Vollmer, M. Optical Properties of Metal Clusters; Springer: Berlin, 1995.

(18) Ricard, D.; Roussignol, P.; Flytzanis, C. SurfaceMediated Enhancement of Optical Phase Conjugation in Metal Colloids. Opt. Lett. 1985, 10, 511.

(19) Collier, C. P.; Saykally, R. J.; Shiang, J. J.; Henrichs, S. E.; Heath, J. R. Reversible Tuning of Silver Quantum Dot Monolayers Through the MetalInsulator Transition. Science 1997, 277, 1978.

(20) Markovich, G.; Collier, C. P.; Heath, J. R. Reversible Metal-Insulator Transition in Ordered Metal Nanocrystal Monolayers Observed by Impedance Spectroscopy. Phys. Rev. Lett. 1998, 80, 3807.

(21) Shiang, J. J.; Heath, J. R.; Collier, C. P.; Saykally, R. J. Cooperative Phenomena in Artificial Solids Made from Silver Quantum Dots: The Importance of Classical Coupling. J. Phys. Chem. B 1998, 102, 3425.

(22) (a) Davis, C. A.; McKenzie, D. R.; McPhedran, R. C. Optical Properties and Microstructure of Thin Silver Films. Opt. Comm. 1991, 85, 70. (b) Rouard, P.; Meesen, A. In Progress in Optics; Wolf, E., Ed.; North-Holland: Amsterdam, 1977; Vol. 15 and references therein.

(23) Mott, N. F. Metal-Insulator Transitions; Taylor \& Francis Ltd.: London, 1990.

(24) Edwards, P. P.; Sienko, M. J. The Transition to the Metallic State. Acc. Chem. Res. 1982, 15, 87.

(25) Heavens, O. S. Optical Properties of Thin Solid Films; Butterworth Scientific Publications: London, 1955; Chapter 4.

(26) Nilsson, P. O. Determination of Optical Constants from Intensity Measurements at Normal Incidence. Appl. Opt. 1968, 435, 7.

(27) Shen, Y. R. Optical Second Harmonic Generation at Interfaces. Annu. Rev. Phys. Chem. 1989, 40, 327.

(28) Takagahara, T. Quantum Dot Lattice and Enhanced Excitonic Optical Nonlinearity. Surf. Sci. 1992, 267, 310.

(29) Remacle, F.; Collier, C. P.; Heath, J. R.; Levine, R. D. The Transition from Localized to Collective Electronic States in a Silver Quantum Dots Monolayer Examined by Nonlinear Optical Response. Chem. Phys. Lett. 1998, 291, 453.

(30) Macdonald, J. R. Impedance Spectroscopy; Wiley: New York, 1987.

(31) Hodge, I. M.; Ingram, M. D.; West, A. R. Impedance and Modulus Spectroscopy of Polycrystalline Solid Electrolytes. J. Electroanal. Chem. 1976, 74, 125. 
(32) Ingram, R. S.; et al. $28 \mathrm{kDa}$ Alkanethiolate-Protected Au Clusters Give Analogous Solution Electrochemistry and STM Coulomb Staircases. J. Am. Chem. Soc. 1997, 119, 9279.

(33) Gerhardt, R. Impedance and Dielectric Spectroscopy Revisited - Distinguishing Localized Relaxation from
Long-Range Conductivity. J. Phys. Chem. Solids 1994, 55, 1491.

(34) Chung, S. W.; Markovich, G.; Heath, J. R. Fabrication and Alignment of Wires in Two Dimensions. J. Phys. Chem. B 1998, 102, 6685.

AR980039X 